\title{
Influência da concentração dos monômeros de acrilato e metacrilato de hidroxipropila na copolimerização em emulsão do estireno com acrilato de $n$-butila e acrilonitrila para produção de adesivos
}

\section{Influence of the hydroxypropyl acrylate and hydroxypropyl methacrylate monomer concentration on the emulsion copolymerization of styrene with $n$-butyl acrylate and acrylonitrile to produce adhesives}

Laura Alves Campos ${ }^{1}$, Samuel A. Santos ${ }^{1}$, Fábio Henrique Franco ${ }^{2}$, Marlon Sandro dos Santos ${ }^{2}$, Lília Müller Guerrini ${ }^{1}$, Maurício Pinheiro de Oliveira ${ }^{1}$

\footnotetext{
${ }^{1}$ Instituto de Ciência e Tecnologia, Universidade Federal de São Paulo-UNIFESP, São José dos Campos, São Paulo, Brasil.

${ }^{2}$ Laboratório de Pesquisa e Desenvolvimento de Polímeros, BASF S.A., Guaratinguetá, São Paulo, Brasil. e-mail: lau_campos@yahoo.com.br, sasantoseq@gmail.com, guerrini@unifesp.br, mauricio.pinheiro@unifesp.br, fabio.franco@basf.com, marlon.santos@basf.com
}

\begin{abstract}
RESUMO
Monômeros acrílicos contendo grupos hidroxila são frequentemente empregados na produção de polímeros, especialmente para aplicações em diferentes substratos ou mesmo em reações posteriores de modificação química com grupos isocianato ou carboxila. Este trabalho tem como objetivo estudar o efeito da concentração dos monômeros de acrilato de hidroxipropila (HPA) e metacrilato de hidroxipropila (HPMA) na copolimerização em emulsão do estireno $(\mathrm{St})$ com o acrilato de $n$-butila $(\mathrm{ABu})$ e acrilonitrila $(\mathrm{ACN})$, visando a obtenção de nanopartículas poliméricas com grupos hidroxila para reações posteriores de modificação química com isocianato. Látexes com diferentes concentrações $(0,3,5,7$ e $10 \%$ m/m) de HPA e HPMA foram obtidos e caracterizados em relação ao teor de sólidos, diâmetro de partícula, distribuição dos tamanhos de partícula, viscosidade, temperatura mínima de formação de filme, estabilidade ao gelo-degelo, concentração de coágulos, dureza do filme e estabilidade coloidal. Os resultados mostram que o aumento da concentração de HPA e HPMA afeta as propriedades físico-químicas dos látexes. Dentre as concentrações de HPMA estudadas, foi possível obter látexes com concentração máxima de 5\% de HPA e 7\% de HPMA. Os látexes produzidos com 10\% de HPA e HPMA apresentaram baixa estabilidade coloidal. Os adesivos foram obtidos via mistura física entre o látex hidroxilado com HPMA e a dispersão de poli(isocianato) cicloalifático contendo $11 \%$ de isocianato bloqueado e caracterizados.
\end{abstract}

Palavras-chave: Polimerização em emulsão, látex hidroxilado, acrilato de hidroxipropila, metacrilato de hidoxipropila, isocianato bloqueado.

\begin{abstract}
Hydroxyl acrylic monomers are important because the hydroxyl group present in the molecule can react with isocyanate groups, conferring better mechanical properties to the final polymer when applied onto some substrates. The aim of this work was to study the effects of the hydroxypropyl acrylate (HPA) and hydroxypropyl methacrylate (HPMA) monomer concentration on the emulsion polymerization of styrene (St) with $n$-butyl acrylate $(\mathrm{ABu})$ and acrylonitrile $(\mathrm{ACN})$. The goal of this work was to obtain latexes with higher concentrations of hydroxyl groups, ideal for reacting with isocyanate, without generating residues or coagulum. Latexes with different amounts of HPA and HPMA (0,3,5,7 e $10 \mathrm{wt} \%)$ were obtained and characterized with respect to solids content, viscosity, particle diameter, particle size distribution, freeze-thaw stability, minimum film forming temperature, coagulum formation, hardness and colloidal stability. The results show that the increased HPMA concentration affected the colloidal properties of the latexes. Among the concentrations of HPMA studied, it was possible to obtain latex with a maximum concentration of 5\% HPA and 7\% HPMA, whereas latex produced with 10\% HPA and HPMA had low colloidal stability.
\end{abstract}


Adhesives was obtained by mixing styrene-acrylic hydroxylated latex with a blocked poly(isocyanate) dispersion and characterized.

Keywords: Emulsion polymerization, hydroxylated latex, hydroxypropyl acrylate, hydroxypropyl methacrylate, blocked isocyanate.

\section{INTRODUÇÃO}

A técnica de polimerização em emulsão é muito empregada para produzir polímeros em meio heterogêneo, empregando monômeros insolúveis e parcialmente solúveis em água, emulsificantes, iniciador e aditivos. A principal vantagem desta técnica de polimerização é a utilização de água ao invés de solventes orgânicos e a obtenção de nanopartículas poliméricas estáveis [1,2]. No entanto, monômeros solúveis em água, como por exemplo, o ácido acrílico, acrilamida e monômeros hidroxilados são tipicamente empregados com a finalidade de melhorar a estabilidade dos látexes, conferir propriedades específicas de aplicação, além de melhorar as propriedades mecânicas dos filmes e acrescentar grupos reativos na cadeia polimérica ou na superfície das nanopartículas de polímero para reações posteriores de modificação química [2-6].

Os monômeros acrílicos hidroxilados ou éster do ácido carboxílico possuem insaturação e função hidroxila na molécula, os quais podem polimerizar via radicais livres na presença ou na ausência de emulsificante [4-10]. Os monômeros hidroxilados mais utilizados na indústria para produção de homopolímeros ou copolímeros são: o acrilato de hidroxietila (HEA), acrilato de hidroxibutila (4-HBA), acrilato de hidroxipropila (HPA) e metacrilato de hidroxipropila (HPMA). As estruturas e as propriedades físico-químicas destes monômeros estão apresentadas na Tabela 1. Os polímeros obtidos a partir destes monômeros são empregados na produção de lentes de contato, géis absorventes, adesivos, agentes de suspensão no tratamento de água, biomateriais, além de apresentarem propriedades de estímulo a resposta em determinados valores de temperatura [8-11]. No entanto, estes monômeros apresentam elevada solubilidade em água, dificultado a utilização dos mesmos na síntese de polímeros via polimerização em emulsão $[4,5,7,8,11]$.

Tabela 1: Estrutura química e propriedades dos monômeros hidroxilados [12].

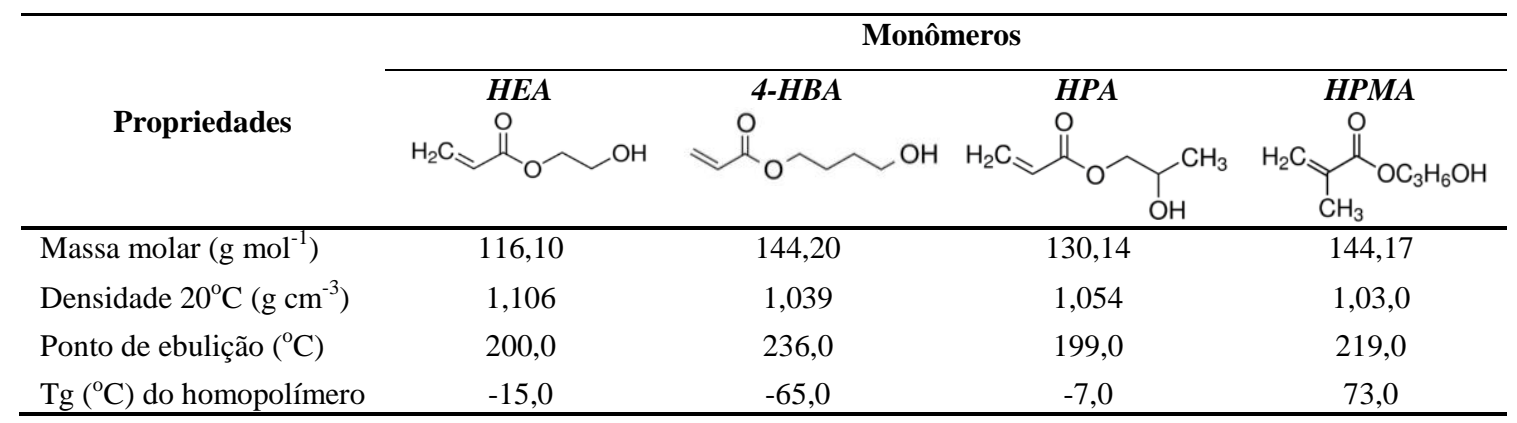

Devido a elevada solubilidade em água destes monômeros, um dos fatores indesejáveis é a polimerização na fase aquosa, com formação de oligômeros e coágulos no látex [5, 6, 11]. Os oligômeros formados são responsáveis por mudanças na viscosidade dos látexes, redução da resistência à água do filme polimérico e principalmente, pela desestabilização durante a síntese e aplicação do látex. Chu e Ou [7] relataram a dificuldade de homopolimerizar monômeros hidroxilados via polimerização em emulsão devido à elevada solubilidade em água destes monômeros, resultando em coagulação e formação de gel durante a polimerização. Mun et al. [9] sintetizaram copolímeros de acrilato de $n$-butila com HEA via polimerização em emulsão. Os autores verificaram que os monômeros possuíam mesma razão de reatividade e o copolímero obtido com $30 \%$ molar de acrilato de $n$-butila apresentou solubilidade em água.

A incorporação de grupos hidroxila, em polímeros obtidos via polimerização em emulsão ou em miniemulsão é muito importante, pois possibilita a preparação de látexes e adesivos dispersos em água, com propriedades superiores de aplicação, para uma vasta gama substratos [13-16]. Sistemas uretânicos híbridos em meio aquoso, constituídos por látex hidroxilado e isocianato são promissores na indústria e possibilitam a eliminação de solventes orgânicos [14,15]. O filme polimérico formado é um sistema hibrido, resultando em filmes poliméricos com boas propriedades de aplicação em diversos tipos de revestimentos. Neste sentido, monômeros acrílicos e pré-polímeros uretânicos podem ser misturados ou polimerizados, para serem empregados em revestimentos para plástico, metais, madeiras, produção de adesivos, tintas decorativas e tintas para impressão $[14,15,20]$. 
A reação dos grupos hidroxila com os grupos isocianato para formar ligações uretânicas, pode ser obtida por meio da mistura do látex hidroxilado com uma dispersão de poli(isocianato) bloqueado, a qual é submetida à temperaturas na faixa de 120 a $250^{\circ} \mathrm{C}$ para que ocorra o desbloqueio dos grupos $\mathrm{NCO}$, seguido pela reação com os grupos hidroxila na superfície das nanopartículas [14,18-22]. Zhang et al. [13] mostraram que a introdução do monômero de HPA na copolimerização do metacrilato de metila para produção de compósitos de poliacrilato com poliuretano via polimerização em emulsão resultou no aumento da resistência a tração do filme polimérico formado. Luz et al. [17] sintetizaram copolímeros de estireno com butadieno na presença do HPMA, em concentrações de 3\% e 5\% visando a substituição do monômero de estireno. De acordo com os autores, o aumento da concentração de HPMA resultou no decréscimo da viscosidade e da massa molar dos copolímeros.

O objetivo deste trabalho foi estudar o efeito da concentração dos monômeros de acrilato de hidroxipropila (HPA) e metacrilato de hidroxipropila (HPMA) na copolimerização em emulsão do St com o $\mathrm{ABu}$ e $\mathrm{ACN}(\mathrm{St} / \mathrm{ABu} / \mathrm{ACN})$, visando a obtenção de nanopartículas hidroxiladas (látexes) com baixa concentração de coágulos para serem empregados na produção de adesivos híbridos (acrílico/poliuretano) base água monocomponente. A influência da concentração e da solubilidade em água dos monômeros hidroxilados nas propriedades dos látexes e dos copolímeros foi avaliada.

\section{MATERIAIS E MÉTODOS}

\subsection{Materiais}

Os monômeros de estireno (St), acrilato de $n$-butila ( $\mathrm{ABu}$ ), acrilonitrila $(\mathrm{ACN})$, acrilato de hidroxipropila (HPA) e metacrilato de hidroxipropila (HPMA) foram obtidos junto à BASF S.A. e utilizados sem purificação prévia, visando representar as condições reais na indústria. Como iniciador de polimerização foi empregado o persulfato de sódio (NaPS, 99,8\%, FMC). Como estabilizante foram utilizados os emulsificantes não-iônico (Emulan ${ }^{\circledR}$ TO 4070, BASF) e aniônico (Disponil ${ }^{\circledR}$ FES 77, BASF). Como agente de transferência de cadeia foi utilizada a $N$-dodecil mercaptana (DDMK, BASF). Para ajuste do pH foi empregada a solução de hidróxido de amônia a $25 \%$ (v/v). Na preparação dos polímeros híbridos, foi empregada a dispersão de poli(isocianato) cicloalifático (Vestanat ${ }^{\circledR}$ EP 10205 E, Evonik), contendo $11 \%$ de grupos NCO bloqueados, $42 \%$ de teor de sólidos, pH igual a 8,9 e viscosidade de $200 \mathrm{mPa}$.s [23]. Em todas as polimerizações foi utilizada água deionizada com condutividade igual a $2,5 \mu \mathrm{S} \mathrm{cm}^{-1}$.

\subsection{Polimerizações}

As polimerizações foram conduzidas em processo semi-contínuo, com diferentes concentrações de HPA e HPMA $(0,3,5,7$ e $10 \%)$ em relação a massa dos monômeros. Foi empregado um reator de vidro encamisado de 1,0 L, equipado com agitador mecânico, condensador de refluxo, termorresistência e banho termostático para aquecimento e resfriamento do reator. No reator, foram adicionados: água deionizada $(106,4 \mathrm{~g})$ e emulsificante aniônico $(1,8 \mathrm{~g})$. A temperatura do meio reacional foi estabilizada para $88^{\circ} \mathrm{C}$ e adicionado $0,3 \mathrm{~g}$ do iniciador NaPS solubilizado em água deionizada. Em seguida, foi iniciada a dosagem da pré-emulsão em $4 \mathrm{~h}$ (Tabela 2). A solução de iniciador com concentração igual a 2,5\% (m/v) $(42,5 \mathrm{~g})$ foi dosada em $4,5 \mathrm{~h}$. Ao final da dosagem do iniciador, a temperatura do meio reacional foi mantida em $88^{\circ} \mathrm{C}$ por $1,0 \mathrm{~h}$ e em seguida, a solução de hidróxido de amônia $(1,0 \mathrm{~g})$ diluída em água deionizada $(13,0 \mathrm{~g})$ foi dosada em $10 \mathrm{~min}$. Ao final, o látex foi resfriado para $35^{\circ} \mathrm{C}$ e filtrado em malha de $0,060 \mathrm{~mm}$.

\subsection{Preparação do adesivo}

Dentre os látexes hidroxilados sintetizados, o látex que apresentou melhor estabilidade e maior concentração de grupos hidroxila foi empregado na aditivação com a dispersão de poli(isocianato) cicloalifático contendo $11 \%$ de isocianato bloqueado. A aditivação foi realizada sob agitação mecânica e rotação de $300 \mathrm{rpm}$. Foi pesado $164 \mathrm{~g}$ do látex hidroxilado e adicionada $236 \mathrm{~g}$ do poli(isocianato) bloqueado. Foi considerada a mesma razão entre o número de equivalentes grama dos grupos isocianato e hidroxila $(\mathrm{NCO} / \mathrm{OH})$. Após adição do poli(isocianato), a mistura ficou sob agitação por $15 \mathrm{~min}$. e em seguida foi filtrada em malha de $0,060 \mathrm{~mm}$. Como forma de comparar os resultados foi realizada a aditivação do látex produzido sem o HPMA. 
Tabela 2: Composições empregadas nas sínteses do poli(St-co-ABu-co-ACN-co-HPA) e poli(St-co-ABu-co-ACN-coHPMA).

\begin{tabular}{lccccc}
\hline \multirow{2}{*}{ Componentes (g) } & \multicolumn{5}{c}{ Polimerizações } \\
\cline { 2 - 6 } & \multicolumn{5}{c}{ Pré-emulsãoros funcionais } \\
\cline { 2 - 6 } & $\mathbf{0 \%}$ & $\mathbf{5 \%}$ & $\mathbf{1 0 \%}$ \\
\hline $\mathrm{H}_{2} \mathrm{O}$ & 120,0 & 120,0 & 120,0 & 120,0 & 120,0 \\
Emulan $^{\circledR}$ TO 4070 & 3,6 & 3,6 & 3,6 & 3,6 & 3,6 \\
Disponil $^{\circledR}$ FES 77 & 9,0 & 9,0 & 9,0 & 9,0 & 9,0 \\
DDMK & 0,9 & 0,9 & 0,9 & 0,9 & 0,9 \\
$\mathrm{St}$ & 176,0 & 176,0 & 176,0 & 176,0 & 176,0 \\
$\mathrm{ABu}$ & 112,0 & 103,0 & 97,0 & 91,0 & 82,0 \\
$\mathrm{ACN}$ & 13,5 & 13,5 & 13,5 & 13,5 & 13,5 \\
$\mathrm{HPA}$ ou HPMA & 0,0 & 9,1 & 15,1 & 21,1 & 30,1 \\
\hline
\end{tabular}

\subsection{Caracterizações}

$\mathrm{O}$ teor de sólidos final dos látexes foi determinado via analise gravimétrica. Uma alíquota $(\approx 1 \mathrm{~g})$ de cada amostra foi pesada em suportes de alumínio e seca em estufa com circulação de ar a $140^{\circ} \mathrm{C}$ por $1 \mathrm{~h}$. A conversão global final foi obtida pela relação entre o teor de sólidos teórico e o teor de sólidos real obtido via análise gravimétrica [24]. $\mathrm{O} \mathrm{pH}$ foi determinado com o auxílio de um pH-metro digital (Micronal, AJX-522). O diâmetro médio das partículas (Dp) e a distribuição dos tamanhos de partículas (DTP) foram determinados por meio da técnica de espalhamento dinâmico de luz (Beckman Coulter, Delsa Nano C). A viscosidade foi determinada em um viscosímetro digital Brookfield, modelo RVT, com fuso 3 e rotação de $20 \mathrm{rpm}$ a $23^{\circ} \mathrm{C}$.

A estabilidade coloidal foi avaliada visualmente e por medidas do diâmetro de partícula, antes e após $72 \mathrm{~h}$ em estufa a $60^{\circ} \mathrm{C}$. A concentração de coágulos (CC) foi determinada via análise gravimétrica. Os látexes foram pesados $(100 \mathrm{~g})$, diluídos em $500 \mathrm{~mL}$ de água deionizada, filtrados com auxílio de uma peneira de aço (malha de $0,125 \mathrm{~mm}$ ) e secos em estufa a $140^{\circ} \mathrm{C}$ por $30 \mathrm{~min}$. [24]. A estabilidade ao gelo-degelo foi determinada a $-5^{\circ} \mathrm{C}$ por $24 \mathrm{~h}$, seguido por $8 \mathrm{~h} \mathrm{a} 25^{\circ} \mathrm{C}$. A temperatura mínima de formação de filme (TMFF) foi determinada sob diferentes valores de temperatura $\left(0\right.$ a $\left.100^{\circ} \mathrm{C}\right)$, em um equipamento da marca Rhopoint Instr., Bar 90, com espessura do filme de $75 \mu \mathrm{m}$.

A dureza dos filmes foi determinada em triplicata via método do pêndulo. Os látexes foram secos com espessura de 75 microns em estufa com circulação de ar a $140^{\circ} \mathrm{C}$, climatizado a $25^{\circ} \mathrm{C}$ por 30 min. e a dureza foi determinada em um durômetro de pêndulo König. As análises de espectroscopia de infravermelho com transformada de Fourier (FT-IR) foram realizadas para identificação dos grupos funcionais no polímero. Foi empregado um espectrofotômetro da Shimadzu via refletância total na faixa de $600 \mathrm{a} 4500 \mathrm{~cm}^{-1}$. As análises de calorimetria exploratória diferencial (DSC) dos copolímeros foram realizadas em um equipamento da marca Netzsch, modelo 204 F1 Phoenix. Os copolímeros foram pesados $(8,0 \mathrm{mg}) \mathrm{em}$ cadinhos de alumínio hermético e as análises foram conduzidas na faixa de temperatura de $30^{\circ} \mathrm{C}$ a $250^{\circ} \mathrm{C}$, com taxa de aquecimento de $10^{\circ} \mathrm{C} \mathrm{min.} .^{-1}$ sob atmosfera de nitrogênio.

\section{RESULTADOS E DISCUSSÕES}

\subsection{Efeito dos monômeros hidroxilados}

As Tabelas 3 e 4 mostram os resultados obtidos para as polimerizações realizadas com diferentes concentrações de HPA e HPMA, respectivamente. Verifica-se que o teor de sólidos e a conversão global final foram afetados pela concentração de HPA e HPMA, quando comparado com o látex na ausência destes monômeros. O efeito da concentração do HPA e HPMA no Dp e na distribuição dos tamanhos de partícula também podem ser vistos nas Tabelas 3 e 4 . A distribuição dos tamanhos de partícula é caracterizada pelo índice de polidispersidade e quanto maior esse valor, mais larga é a distribuição. Com a introdução de 3\% de HPA o diâmetro das partículas e a distribuição dos tamanhos de partículas aumentaram para $241 \mathrm{~nm}$ e 0,153 respectivamente, indicando a formação de partículas com maior diâmetro e mais polidispersas. Para a polimerização conduzida com 5\% de HPA, o diâmetro de partícula diminuiu para $120 \mathrm{~nm}$ e a polidispersão aumentou para 0,193 indicando a formação de um número maior partículas com menor diâmetro. Já as polimerizações conduzidas com 7 e $10 \%$ de HPA ocorreu coagulação dos látexes antes do final das polimerizações, não sendo possível quantificar o coágulo formado. Estes resultados indicam que a coagulação dos látexes está relacionada com a formação de partículas pequenas, as quais coagulam por falta 
de estabilizante no meio reacional. Assim, para a composição empregada a concentração limite de HPA está abaixo de $5 \%$ para produção de copolímeros de poli(St-co-ABu-co-ACN-co-HPA).

Tabela 3: Propriedades coloidais dos látexes de St/ABu/ACN/HPA com diferentes concentrações de HPA.

\begin{tabular}{lccccc}
\hline \multirow{2}{*}{\multicolumn{1}{c}{ Propriedades }} & \multicolumn{5}{c}{ Látexes } \\
\cline { 2 - 6 } & $\mathbf{0 \% H P A}$ & $\mathbf{3 \% H P A}$ & $\mathbf{5 \% H P A}$ & $\mathbf{7 \% H P A}$ & $\mathbf{1 0 \% H P A}$ \\
\hline Teor de sólidos (\%) & 48,7 & 48,8 & 50,80 & 20,5 & -- \\
Conversão global (\%) & 95,4 & 95,6 & 99,5 & 40,1 & -- \\
Diâmetro de partícula (nm) & 165,4 & 241,0 & 120,0 & -- & -- \\
Polidispersão & 0,086 & 0,153 & 0,193 & -- & -- \\
pH & 9,5 & 9,4 & 9,2 & -- & -- \\
Viscosidade (mPa.s) & 1250 & 1400 & 1450 & -- & -- \\
Coágulos (ppm) & 120 & 200 & 350 & -- & -- \\
Estabilidade ao gelo-degelo (ciclos) & 3 & 3 & 2 & -- & -- \\
Estabilidade visual & OK & OK & OK & -- & -- \\
Dp após 72h a 60 ${ }^{\circ} \mathrm{C}(\mathrm{nm})$ & 176,0 & 260,4 & 140,0 & -- & -- \\
TMFF $\left({ }^{\circ} \mathrm{C}\right)$ & 68,0 & 45,0 & 40,0 & -- & -- \\
Dureza (ciclos) & $74( \pm 3)$ & $65( \pm 5)$ & $58( \pm 4)$ & -- & - \\
\hline
\end{tabular}

Tabela 4: Propriedades coloidais dos látexes St/ABu/ACN/HPMA com diferentes concentrações de HPMA.

\begin{tabular}{lccccc}
\hline \multirow{1}{*}{ Propriedades } & \multicolumn{5}{c}{ Látexes } \\
\cline { 2 - 6 } & O\%HPMA & $\mathbf{3 \% H P M A}$ & $\mathbf{5 \% H P M A}$ & $\mathbf{7 \% H P M A}$ & $\mathbf{1 0 \%} \boldsymbol{H P M A}$ \\
\hline Teor de sólidos (\%) & 50,83 & 50,6 & 50,7 & 50,5 & 45,7 \\
Conversão global (\%) & 99,5 & 99,1 & 99,3 & 98,8 & 89,5 \\
Diâmetro de partícula (nm) & 147,3 & 134,2 & 114,5 & 129,1 & 197,4 \\
Polidispersão & 0,073 & 0,013 & 0,190 & 0,198 & 0,186 \\
pH & 9,6 & 9,7 & 9,4 & 9,4 & 9,5 \\
Viscosidade (mPa.s) & 1250 & 1400 & 1450 & 1750 & 3500 \\
Coágulos (ppm) & 81,6 & 115,8 & 141,9 & 172,8 & 1472,6 \\
Estabilidade ao gelo-degelo (ciclos) & 3 & 3 & 3 & 3 & 0 \\
Estabilidade visual & $\mathrm{OK}$ & $\mathrm{OK}$ & $\mathrm{OK}$ & $\mathrm{OK}$ & Ruim \\
Dp após 72h a 60 ${ }^{\circ} \mathrm{C}(\mathrm{nm})$ & 140,0 & 135,1 & 134,5 & 130,0 & 182,8 \\
TMFF $\left({ }^{\circ} \mathrm{C}\right)$ & 66,0 & 64,7 & 62,3 & 57,11 & 31,3 \\
Dureza (ciclos) & $75( \pm 4)$ & $94( \pm 3)$ & $107( \pm 2)$ & $118( \pm 4)$ & $90( \pm 3)$ \\
\hline
\end{tabular}

Para as polimerizações conduzidas com HPMA (Tabela 4), verifica-se um pequeno decréscimo do diâmetro de partícula para os látexes obtidos com 5\% e 7\% de HPMA em comparação com o diâmetro de partícula dos látexes com $0 \%$ e $3 \%$ de HPMA. No entanto, a polidispersão também aumentou com a concentração de HPMA. Os látexes com 0\% e 3\% de HPMA apresentaram polidispersão de 0,073 e 0,013 respectivamente, enquanto que os látexes com 5\% e 7\% apresentaram polidispersão de 0,190 e 0,198. É importante ressaltar, que o látex produzido com $10 \%$ de HPMA apresentou diâmetro de partícula e polidispersão superiores ao diâmetro e a polidispersão do látex produzido com $0 \%$ de HPMA. A concentração de coágulos para o látex com 10\% de HPMA foi igual a 1472,6 ppm, ou seja, 18 vezes maior em comparação com a concentração obtida para o látex sem a introdução do HPMA. A Figura 2 mostra a distribuição dos tamanhos de partícula em triplicata para os látexes com $0 \%$ e $10 \%$ de HPMA. 

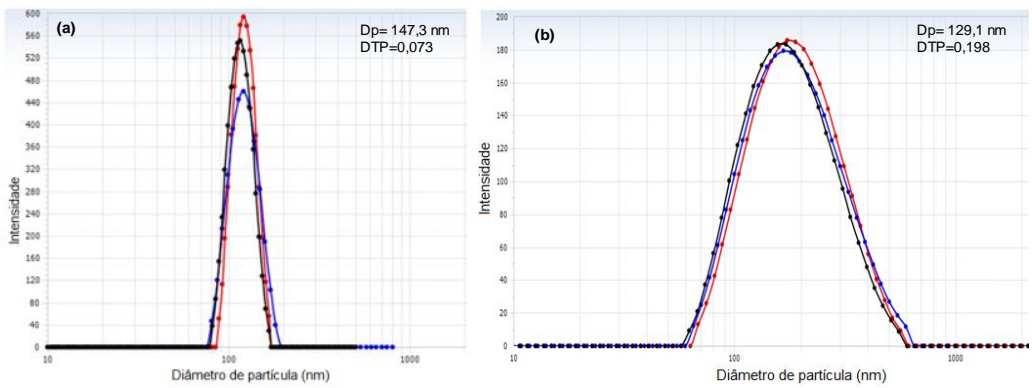

Figura 1: Distribuição dos tamanhos de partícula (DTP) dos látexes com 0\% de HPMA (a) e 10\% de HPMA (b).

Comparando os resultados do diâmetro médio de partícula dos látexes obtidos, verifica-se que o HPMA teve menor influência no diâmetro de partícula, porém, a polidispersão também aumentou para os látexes produzidos com a concentração de HPMA superior a 5\%. Estes resultados estão relacionados à solubilidade em água dos monômeros de HPA e HPMA, além do tipo de iniciador e agente de transferência de cadeia empregados [4-8,26]. Como o HPMA possui menor solubilidade em água em relação ao HPA, ele polimeriza em maior proporção na fase orgânica e nas partículas de polímero, enquanto que o HPA polimeriza em maior proporção na fase aquosa, via nucleação homogênea, seguida por coagulação das partículas formadas [4-7]. No decorrer da polimerização, a quantidade de emulsificante empregada não foi suficiente para estabilizar todas as partículas formadas, resultando em perda de estabilidade, com aumento da polidispersão e por consequiência, formação de coágulos via mecanismo de heterocoagulação. A perda de estabilidade na reação com 7 e 10\% de HPA pode ser explicada pela formação de um número maior de partículas de menor diâmetro, muito provavelmente pela maior solubilidade em água do HPA, resultando na desestabilização das partículas. Ali et al. [11] observaram o aumento do diâmetro de partícula e redução da concentração de coágulos de $9 \%$ para $6 \%$ com a introdução de acrilato de etila como comonômero.

De acordo com os resultados obtidos neste trabalho, é possível confirmar a hipótese do efeito da solubilidade em água do HPMA (10\% v/v) na etapa de nucleação das partículas [26]. Conforme descrito por Chu e Fu [4] o HPMA em água forma uma solução homogênea somente na relação de 1 parte de HPMA para 9 partes de água. Com o aumento da concentração de HPMA ou HPA ocorre a formação de um número maior de partículas via nucleação homogênea com menor diâmetro e maior polidispersão. Assim, com o aumento da concentração de HPMA ou HPA, a polimerização ocorre preferencialmente na fase aquosa até atingir um tamanho crítico " $z$ " $\left(\mathrm{RM}_{z}^{*}\right)$, podendo adicionar novas moléculas de HPA ou HPMA até atingir o tamanho crítico, $J_{\text {crit. }}$, e formar novas partículas via nucleação homogênea. Os radicais $\mathrm{RM}^{*}{ }_{i}$ formados podem transferir cadeia na fase aquosa ou terminar nesta mesma fase com radicais primários e com radicais oligoméricos resultando no aumento da concentração de coágulos [2,4-7]. Kamei et al.[8] mostraram que a taxa de polimerização em emulsão do St com HEMA é maior em comparação com a taxa de polimerização do St devido a formação de partículas secundárias via nucleação homogênea. De acordo com Hoogenboom et al. [10] o grupo metil na molécula do HPMA não tem influência na cinética de polimerização do HPMA e do HEA, apresentando mesmo comportamento cinético.

Nas Tabelas 3 e 4 verifica-se o efeito do HPA e do HPMA na viscosidade e na estabilidade dos látexes a $60^{\circ} \mathrm{C}$. Com a introdução do HPA e do HPMA verifica-se um pequeno aumento da viscosidade dos látexes em comparação com a viscosidade dos látexes sem os monômeros hidroxilados. $\mathrm{O}$ aumento da viscosidade foi mais significativo para os látexes obtidos com 7 e $10 \%$ de HPMA. Os látexes após $72 \mathrm{~h}$ em estufa a $60^{\circ} \mathrm{C}$ apresentaram-se estáveis para as concentrações de até 5\% de HPA e 7\% de HPMA. O diâmetro de partícula após $72 \mathrm{~h} \mathrm{a} 60^{\circ} \mathrm{C}$ permaneceu praticamente constante, sem o incremento da viscosidade e separação de fases. No entanto, o látex produzido com 10\% de HPMA apresentou baixa estabilidade e separação de fases após $72 \mathrm{~h} \mathrm{a} 60^{\circ} \mathrm{C}$. A estabilidade ao congelamento e descongelamento foi igual a 3 ciclos para os látexes com 0, 3, 5 e 7\% de HPMA, enquanto que o látex produzido com 10\% de HPMA não apresentou estabilidade, coagulando no primeiro ciclo de congelamento e descongelamento. Para os látexes produzidos com 0 e $3 \%$ de HPA a estabilidade ao congelamento e descongelamento foi igual a 3 ciclos, enquanto que para o látex com 5\% de HPA foi igual a 2 ciclos. Sedlak, et al. [22] relataram que a formação de partículas poliméricas estáveis com diâmetro de $100 \mathrm{~nm}$ contendo HEMA requer a escolha correta do iniciador, emulsificante e balanço hidrofílico/hidrofóbico apropriado entre os componentes da formulação.

$\mathrm{Na}$ Tabela 3 também é possível verificar um decréscimo da TMFF e da dureza dos filmes poliméricos com o aumento da concentração do monômero de HPA, muito provavelmente pela menor Tg do poli(HPA) que é de aproximadamente $-7,0^{\circ} \mathrm{C}$. Já para os látexes produzidos com o HPMA (Tabela 4), a TMFF e a 
dureza aumentaram com o aumento da concentração do HPMA. Para o látex produzido com 7\% de HPMA a TMFF foi igual a $57^{\circ} \mathrm{C}$ e dureza de 118 ciclos, enquanto que para o látex sem HPMA a TMFF foi igual a $66{ }^{\circ} \mathrm{C}$ e a dureza igual a 75 ciclos. Estes resultados podem ser justificados pela Tg do poli(HPMA) e pela formação de oligômeros na fase aquosa, ricos em HPMA, resultando no aumento mais significativo da dureza. Considerando que a $\mathrm{Tg}$ do poli(HPMA) está entre 73 e $76^{\circ} \mathrm{C}[12,27]$ e a solubilidade em água do HPMA é maior que $13 \% \mathrm{~m} / \mathrm{m}$ [11], o poli(HPMA) formado na fase aquosa pode atuar como coalescente, alterando a TMFF e aumentando a dureza dos filmes.

\subsection{Propriedades dos adesivos}

Com base nos resultados apresentados nas Tabelas 3 e 4, o látex com as melhores propriedades foi obtido com a concentração de 7\% de HPMA por apresentar maior concentração de HPMA e menor concentração de coágulos. O látex com 10\% de HPMA não apresentou boa estabilidade coloidal devido ao elevado teor de coágulos (Figura 2). Para efeito de comparação foi escolhido o látex sem HPMA para ser aditivado com a dispersão de poli(isocianato) bloqueado. A Tabela 5 mostra os resultados obtidos empregando os dois látexes ( 0 e 7\% de HPMA) aditivados com a dispersão de poli(isocianato) bloqueado. A estabilidade e as propriedades coloidais são fortemente influenciadas pelo diâmetro e distribuição do diâmetro das partículas, além da densidade de grupos $\mathrm{OH}$ na superfície e ou na fase aquosa, em função da natureza hidrofílica do polímero formados nesta fase.

Tabela 5: Propriedades físico-químicas dos adesivos preparados com a dispersão de poli(isocianato) bloqueado.

\begin{tabular}{lcc}
\hline & \multicolumn{2}{c}{ Adesivos } \\
\cline { 2 - 3 } \multicolumn{1}{c}{ Propriedades } & O\%HPMA/PU & $\mathbf{7 \% H P M A / P U}$ \\
\hline Teor de sólidos (\%) & 45,6 & 45,6 \\
Viscosidade (mPa.s) & 75,0 & 50,0 \\
Diâmetro de partícula (nm) & 114,8 & 148,8 \\
Polidispersão & 0,074 & 0,076 \\
pH & 9,6 & 10,0 \\
Dureza (ciclos) & 55,0 & 67,0 \\
TMFF $\left({ }^{\circ} \mathrm{C}\right)$ & 75,0 & 68,0 \\
Coágulos (ppm) & 29,92 & 46,93 \\
Estabilidade $\left(60^{\circ} \mathrm{C} / 72 \mathrm{~h}\right)$ & OK & OK \\
Estabilidade ao gelo-degelo & 1 ciclo & 1 ciclo \\
(ciclos) & & \\
\hline
\end{tabular}

Na Tabela 5 verifica-se que a introdução da dispersão de poli(isocianato) bloqueado e a presença de hidroxilas no látex não teve influência na estabilidade dos adesivos. A estabilidade das misturas foi verificada visualmente pela ausência de sedimentação após $72 \mathrm{~h}$ a $60^{\circ} \mathrm{C}$. $\mathrm{O} \mathrm{pH}$ se manteve próximo aos valores obtidos sem a adição da dispersão de poli(isocianato). $\mathrm{O}$ diâmetro de partícula e a polidispersão dos látexes aditivados apresentaram pequena variação, quando comparado ao diâmetro dos látexes sem a introdução da dispersão de poli(isocianato).

Independente da concentração de HPMA no látex a viscosidade dos látexes aditivados com a dispersão de poli(isocianato) foi menor e pode ser explicada pela baixa viscosidade da dispersão de poli(isocianato) bloqueado (200 $\mathrm{mPa} . \mathrm{s})$ e pela interação entre as partículas do látex com as partículas da dispersão de poli(isocianato). $\mathrm{O}$ aumento da razão $\mathrm{NCO} / \mathrm{OH}$ facilita a dispersão, porém afeta a estabilidade ao delo-degelo das dispersões. A TMFF dos látexes aumentou após a introdução da dispersão de poli(isocianato). A $\mathrm{Tg}$ do poli(isocianato) bloqueado é inferior a $10^{\circ} \mathrm{C}$ [23]. Como a dispersão de poli(isocianato) não apresentava coágulos, foi verificada baixa concentração de coágulos nos adesivos (Tabela 5). A dureza dos filmes (Tabela 4 e 5) está relacionada com a concentração de poli(isocianato) empregada na preparação dos adesivos e a concentração de grupos hidroxila no latex. Com a introdução da dispersão de poli(isocianato) no látex sem hidroxila (0\%HPMA) a dureza reduziu de 75 ciclos para 55 ciclos. Já para o filme preparado com o látex contendo 7\% de HPMA e a dispersão de poli(isocianato) a dureza caiu de 118 ciclos para 68 ciclos. Estes resultados indicam que a dispersão de poli(isocianato) atua como plastificante, reduzindo a dureza e a TMFF do adesivo preparado com o látex hidroxilado.

A Figura 3(a-c) mostra os espectros FT-IR dos polímeros com 0\%HPMA (a), 7\%HPMA (b) e após aditivação com a dispersão de poli(isocianato) (c). As bandas características dos grupos hidroxila $(\mathrm{OH})$, carbonila $(\mathrm{C}=\mathrm{O})$ e isocianato $\left(\mathrm{N}=\mathrm{C}=\mathrm{O}\right.$ ), são atribuídas, respectivamente, em $\approx 3400 \mathrm{~cm}^{-1}, \approx 1720 \mathrm{~cm}^{-1} \mathrm{e} \approx 2270$ $\mathrm{cm}^{-1}[11,17,18,28]$. Verifica-se que o polímero sem a introdução do HPMA (Figura 3a) não apresenta o pico 
referente ao estiramento $\mathrm{OH}$ na região de $\approx 3400 \mathrm{~cm}^{-1}$, enquanto que o espectro apresentado na Figura $3 \mathrm{~b}$ apresenta um pico mais largo, associado ao estiramento do grupo $\mathrm{OH}$ oriundo do HPMA, indicando a presença da hidroxila do HPMA na cadeia polimérica. Também é possível observar a banda característica da carbonila de éster na região de $1735-1750 \mathrm{~cm}^{-1}$. Com a introdução do poli(isocianato) bloqueado, verifica-se em $2270 \mathrm{~cm}^{-1}$ a presença do isocianato que não reagiu com a hidroxila e a água (Figura 3c).
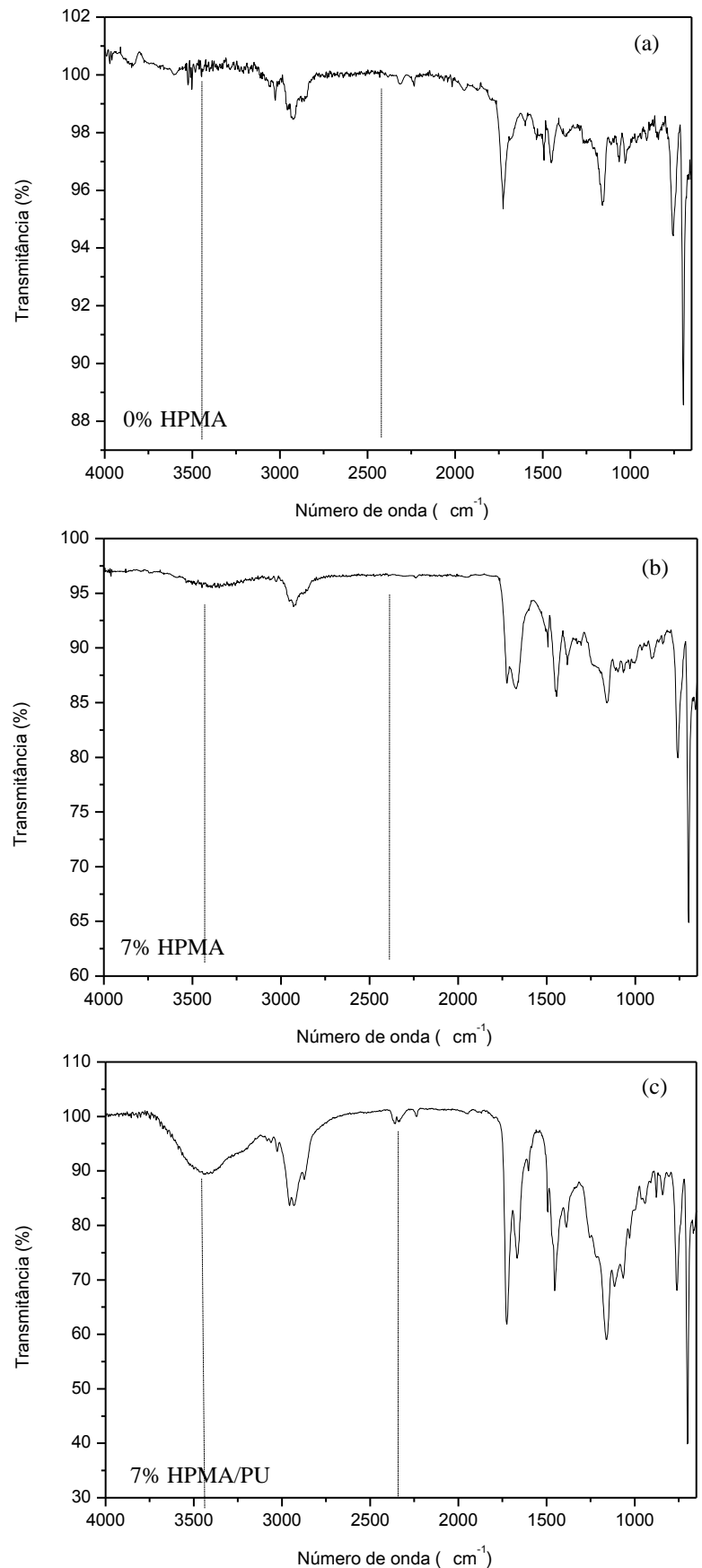

Figura 2: Análises de FT-IR dos polímeros com 0\% de HPMA (a), 7\% de HPMA (b) e 7\% de HPMA aditivado com poli(isocianato) (c).

Para entender o efeito da introdução do poli(isocianato) bloqueado na estrutura do polímero e o desbloqueio mediante aquecimento, foi estudado o comportamento térmico do adesivo via calorimetria exploratória diferencial. A presença de um pico endotérmico está relacionada ao desbloqueio do isocianato e reações entre os grupos hidroxila e o isocianato [18,30]. A Figura 3a,b mostra as curvas de DSC para os adesivos com e sem HPMA. De acordo com os resultados é possível verificar que ocorreu desbloqueio do isocianato durante o aquecimento, seguido por reações de reticulação, muito provavelmente em função das diferentes intensidades dos picos. Para o adesivo formulado com poli(isocianato) e o látex sem HPMA 
(Figura 3a) a fusão teve início em aproximadamente $150^{\circ} \mathrm{C}$, com máximo em $185^{\circ} \mathrm{C}$, apresentando um pico mais largo e profundo. Já para o polímero com 7\% de HPMA (Figura 3b) o pico de fusão foi menos intenso, iniciando em aproximadamente $175^{\circ} \mathrm{C}$ e máximo em $200^{\circ} \mathrm{C}$. Estes comportamentos indicam a formação de reações de reticulação, as quais necessitaram de maior energia para romper as ligações formadas entre os grupos hidroxila e o isocianato. De acordo com a literatura [23] o desbloqueio do poli(isocianato) ocorre na faixa de temperatura entre 140 a $150^{\circ} \mathrm{C}$. Carlson et al. [29] descreve que o desbloqueio do isocianato via temperatura pode formar estruturas de alofanato. $\mathrm{Na}$ faixa de temperaturas entre 90 e $110^{\circ} \mathrm{C}$, verifica-se uma pequena inflexão indicando (pontilhado) a Tg dos polímeros estudados. No entanto, não é possível afirmar que a Tg dos polímeros está nesta faixa de temperatura, uma vez que os valores da TMFF (Tabela 5) estão distantes desta faixa de temperatura.
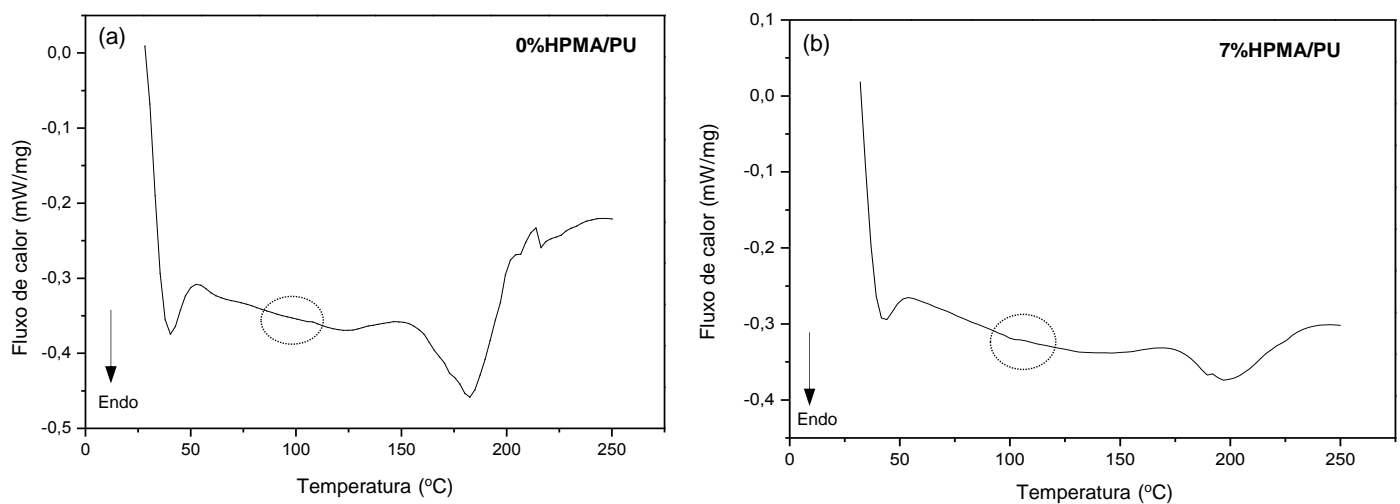

Figura 3: DSC dos adesivos preparados com poli(isocianato) com os látexes: (a) sem HPMA e (b) com 7\% de HPMA.

\section{CONCLUSÕES}

De acordo com os resultados obtidos conclui-se que o aumento da concentração de HPA e HPMA nas polimerizações em emulsão do estireno com acrilato de n-butila e acrilonitrila afeta as propriedades físicoquímicas dos látexes. Foi observado um aumento na viscosidade, na concentração coágulos e na dureza do filme polimérico com o aumento da concentração de HPA e HPMA. Conclui-se também que é possível obter látexes estáveis e com baixa concentração de coágulos para a concentração limite de 7\% de HPMA na formulação. Dentre as concentrações de HPMA estudadas, foi possível obter látexes estáveis com a concentração máxima de 5\% de HPA e 7\% de HPMA, enquanto que os látexes produzidos com 7 e $10 \%$ HPA apresentaram baixa estabilidade coloidal durante a polimerização. Acima da concentração limite, foi observado o inverso, baixa dureza do filme, baixa TMFF, alta viscosidade e baixa estabilidade ao congelamento e descongelamento. A incorporação da dispersão de poli(isocianato) contendo $11 \%$ de isocianato bloqueado afetou as propriedades físico-químicas dos látexes, resultando na redução da viscosidade do adesivo, redução da TMFF, decréscimo da dureza dos filmes poliméricos e baixa estabilidade ao congelamento e descongelamento. Látexes estáveis à temperatura de $60^{\circ} \mathrm{C}$ foram obtidos com a introdução da dispersão de poli(isocianato). Reações de reticulação do tipo $\mathrm{NCO} / \mathrm{OH}$ foram identificadas via análise de DSC e FT-IR, confirmando a reação entre as hidroxilas no látex polimérico com os grupos NCO do poli(isocianato).

\section{AGRADECIMENTOS}

Os autores agradecem a FAPESP (processo 2013/25619-3) e a BASF S.A. pela bolsa concedida, doação dos reagentes e caracterizações.

\section{BIBLIOGRAFIA}

[1] GILBERT, R.G., Emulsion Polimerization: A Mechanistic Approach, London, Academic Press, 1995.

[2] OLIVEIRA, M.P., GIORDANI, D.S., SANTOS, A.M., "The role of itaconic and fumaric acid in the emulsion copolymerization of methyl methacrylate and n-butyl acrylate", European Polymer Journal, v. 42, pp. 1196-1205, 2006.

[3] VORWERG, L., GILBERT, R.G.,"Electrosteric stabilization with poly(acrylic) acid in emulsion polymerization: Effect on kinetics and secondary particle formation”, Macromolecules, v. 33, pp. 6693-6703, 2000 .

[4] CHU, H.H., FU, D.C., "Preparation of poly(hydroxyethy1 methacrylate) and poly(hydroxypropy1 
methacrylate) latices", Macromolecules Rapid Communication, v. 19, pp. 107-110, 1998.

[5] TAUER, K., IMROZ ALI, A.M., YILDIZ, U., et al., "On the role of hydrophilicity and hydrophobicity in aqueous heterophase polymerization", Polymer, v. 46, pp. 1003-1015, 2005.

[6] GILBERT, R.G., FEENEY, P.J., NAPPER, D.H., "Surfactant-free emulsion polymerizations-predictions of coagulative nucleation theory", Macromolecules, v. 20, pp. 2922-2930, 1987.

[7] CHU, H.H., OU, E.D. "Emulsion polymerization of 2-hydroxyethyl methacrylate and partition of monomer between particles and water phase", Polymer Bulletin, v. 44, pp. 337-344, 2000.

[8] KAMEI, S., OKUBO, M., MATSUMOTO, T. "Production of anomalous particles in the process of emulsifier-free emulsion copolymerization of styrene and 2-hydroxyethyl methacrylate", Journal of Polymer Science: Part A: Polymer Chemistry, v. 24, pp. 3109-3116, 1986.

[9] MUN, G.A., NURKEEVA, Z.S., BEISSEGUL, A.B., et al., "Temperature-Responsive water-soluble copolymers based on 2-hydroxyethyl acrylate and butyl acrylate", Macromolecular Chemistry Physics, v. 208, pp. 979,987, 2007.

[10] HOOGENBOOM, R., POPESCU, D., STEINHAUER, W., et al., "Nitroxide-mediated copolymerization of 2-hydroxyethyl acrylate and 2-hydroxypropyl acrylate: Copolymerization kinetics and thermoresponsive properties", Macromolecular Rapid Communications, v.30, pp. 2042-2048, 2009

[11] ALI, A.M.I., PAREEK, P., SEWELL, L., et al., "Synthesis of poly(2-hydroxypropyl methacrylate) latex particles via aqueous dispersion polymerization", Soft Matter, v. 3, pp.1003-1013, 2007.

[12] BASF Specialty Monomers, Technical Information,

http://www.specialty-monomers.basf.com/portal/streamer?fid=235715. Acessado junho de 2017.

[13] ZHANG, H.T., GUAN, R., YIN, Z.H., LIN, L.L., "Soap-free seeded emulsion copolymerization of MMA onto PU-A and their properties”, Journal of Applied Polymer Sci., v. 82, pp. 941-947, 2001.

[14] COUTINHO, F.M.B., DELPECH, M.C., "Poliuretanos como materiais de revestimento de superfície" Polímeros: Ciência e Tecnologia, v. 6, pp. 41-48, 1999.

[15] COUTINHO, F.M.B., DELPECH, M.C., SANTOS, C.C., et al., "Síntese e caracterização de dispersões aquosas de poliuretanos à base de copolímeros em bloco de poli(glicol etilênico) e Poli(glicol propilênico)", Química Nova, v. 31, pp. 1437-1443, 2008.

[16] ZHANG, J., XUEFENG, L., XINHAO, S., et al., "Synthesis of core-shell acrylic-polyurethane hybrid latex as binder of aqueous pigment inks for digital inkjet printing", Progress in Natural Science: Materials Internacional, v. 22, pp. 71-78, 2012.

[17] LUZ, R.F., FURTADO, C.R.G., SOARES, B.G., "Obtenção de copolímero de butadieno e estireno funcionalizado com metacrilato de hidroxipropila", In: $11^{0}$ Congresso Brasileiro de Polímeros, Campos do Jordão, pp. 3559-3565, Campos do Jordão, 2011.

[18] MIRCEVA, A., JANEŽIC, M., ŽIGON, M., MALAVAŠIC, T., "Characterization of blocked isocyanates", Journal of Molecular Structure, v. 267, pp. 129-134, 1992.

[19] WICKS, Z.W., "Coatings" em "Encyclopedia of Polymer Science and Engineering", Supp. MARK, H.F., BIKALES, N.M. OVERBERGER, C.G., MENGES, G. Ed. John Wiley and Sons, Inc., New York 1990.

[20] HEGEDUS, C.R., KLOIBER, K.A., Journal of Coatings Technology Research, v.68, pp. 39-48 1996.

[21] FRISCH, K.C., XIAO, H.X., CZERWINSKI, R.W., Adhesives Age, pp. 41-54, 1988.

[22] YANG, H., MENDON, S. K., S. K. AWLINS, S. K. "Nanoencapsulation of blocked isocyanates through aqueous emulsion polymerization", eXPRESS Polymer Letters, v.2, pp. 349-356, 2008.

[23] EVONIK INDUSTRIES, EP-DS1205, http://coatings.panpage.de/En/Crosslinkers/VESTANAT/VESTANAT_nicht_veroeffentlichen/VESTANAT_ EP_DS_1205_e.pdf Acessado junho de 2018.

[24] OLIVEIRA, M.P., SILVA, C.R. GUERRINI, L.M., Journal of Coatings Technology Research, v.8, pp.439-447, 2011.

[25] LEDUC, E.H., HOLT, S.J., "Hydroxypropyl methacrylate, a new water-miscible embedding medium for electron microscopy", Journal of Cell Biology, v. 26, pp. 137-155, 1965.

[26] ALI, A.M.I., TAUER, K., SEDLAK, M. "Comparing emulsion polymerization of methacrylatemonomers with different hydrophilicity”, Polymer, v.46, pp. 1017-1023, 2005. 
[27] BRANDRUP, J., IMMERGUT, E.H. Polymer Handbook, $2^{\text {nd }}$ Ed., John Wiley \& Sons, Inc., New York, 1975.

[28] PEROVA, T.S., VIJ, J.K., XU, H. "Fourier transform infrared study of poly(2-hydroxyethyl methacrylate) PHEMA", Colloid \& Polymer Science, v. 275, pp. 323-332, 1997.

[29] CARLSON, G.M., NEAG, C.M., KUO, C., PROVDER, T. "Fourier-Transform Infrared Characterization of Polymers", Plenum Press, New York, pp.197, 1987.

[30] LEE, J.M., SUBRAMANI, S., LEE, Y.S., KIM, J.H. "Thermal decomposition behavior of blocked diisocyanates derived from mixture of blocking agents”, Macromolecular Research, v. 13, pp. 427-434, 2005.

\section{ORCID}

Marlon Sandro dos Santos Maurício Pinheiro de Oliveira

Samuel Augusto dos Santos

Lília Müller Guerrini

Laura Alves Campos

Fábio Henrique Franco https://orcid.org/0000-0002-7810-7647

https://orcid.org/0000-0002-8210-5134

https://orcid.org/0000-0003-0100-1219

https://orcid.org/0000-0001-8824-2968

https://orcid.org/0000-0003-4341-7044

https://orcid.org/0000-0003-0889-6641 